\title{
Immune checkpoint inhibitors combined with chemotherapy/ bevacizumab therapy for patients with advanced lung cancer and heavily treated with EGFR mutation: a retrospective analysis
}

\author{
Ran Hu", Zhiting Zhao", Yue Shi, Meiqi Shi, Guohao Xia, Shaorong Yu, Jifeng Feng \\ Department of Medical Oncology, The Affiliated Cancer Hospital of Nanjing Medical University \& Jiangsu Cancer Hospital \& Jiangsu Institute of \\ Cancer Research, Nanjing, China \\ Contributions: (I) Conception and design: S Yu; (II) Administrative support: J Feng; (III) Provision of study materials or patients: R Hu; (IV) \\ Collection and assembly of data: R Hu; (V) Data analysis and interpretation: Z Zhao; (VI) Manuscript writing: All authors; (VII) Final approval of \\ manuscript: All authors. \\ "These authors contributed equally to this work. \\ Correspondence to: Shaorong Yu; Jifeng Feng. Baiziting No.42, Nanjing 210009, China. Email: yushaorong2009@163.com; jifeng_feng@163.com.
}

Background: EGFR-mutated lung cancer poorly responded to anti-programmed death-1 (PD-1)/ programmed death-ligand 1 (PD-L1) monotherapy. Whether patients with EGFR-mutated lung cancer can benefit from anti-PD-1/PD-L1 therapy combined with other drugs remains controversial. We retrospectively evaluated the safety and efficacy of the PD-1 inhibitor combined with other drugs (chemotherapy and/ or bevacizumab) in patients with EGFR-mutated lung cancer, who have progressed on EGFR-TKI treatment to determine the activity of the anti-PD-1/PD-L1 therapy combined with chemotherapy or/and bevacizumab therapy in heavily treated patients with EGFR-mutated lung cancer.

Methods: We identified 56 patients with EGFR-mutated lung cancer treated with PD-1/PD-L1 inhibitors alone or combined with the chemotherapy/bevacizumab therapy. The objective response rates were assessed using RECIST v1.1. Adverse events (AEs) were graded in accordance with the National Cancer Institute Common Terminology Criteria for Adverse Events v4.0. The study was conducted in accordance with the Declaration of Helsinki (as revised in 2013). The study was approved by the Academic Ethics Committee of Jiangsu Cancer Hospital. (NO. 2019 160), and individual consent for this retrospective analysis was waived.

Results: Objective responses were observed in 6 of $56(10.7 \%)$ patients, and the disease control rate was 53.6\% (30/56). The median progression-free survival (PFS) was 3.33 months with $95 \%$ CI of $1.58-5.08$ months. No patient achieved a complete response. All six patients that achieved PR were treated with the PD-1 inhibitor combined with chemotherapy or bevacizumab therapy. Three of the six patients who achieved PR were treated with radiotherapy combined with PD-1 inhibitor-based therapy. Patients treated with the PD-1 inhibitor-based therapy as second-line therapy showed relatively longer PFS and higher objective response rates than those treated with PD-1 inhibitor-based therapy as third- or late-line therapy (PFS: 5.50 vs. 3.27 months, $\mathrm{P}=0.301$; objective response rates: $25.0 \%$ vs. $6.82 \%, \mathrm{P}=0.071$ ). No additional AE profile was observed.

Conclusions: The PD-1 inhibitor combined with the chemotherapy/bevacizumab therapy showed acceptable toxicity profile and moderate efficacy on heavily treated advanced EGFR-mutated lung cancer after the exhaustion of target therapy.

Keywords: EGFR mutation; lung adenocarcinoma; programmed death-1 (PD-1); chemotherapy

Submitted Dec 15, 2020. Accepted for publication Apr 02, 2021.

doi: $10.21037 /$ jtd-20-3520

View this article at: http://dx.doi.org/10.21037/jtd-20-3520 


\section{Introduction}

Immune checkpoint inhibitors, especially anti-programmed death-1 (PD-1)/programmed death-ligand 1(PD-L1) antibody, serve as new standard of care for patients with advanced lung cancer without oncogenic driver alternation because of their efficacy and relatively low toxicity (1-3). The EGFR-mutated lung cancer is one of the most important oncogenic driver mutations in patients with lung cancer. Approximately $50 \%$ of patients with lung adenocarcinoma in Asia develops active EGFR mutation. The target therapy proves excellent efficacy in advanced lung cancer with EGFR mutation having $~ 70 \%$ response rate $(4,5)$. However, almost all patients eventually develop resistance to the target therapy after treatment. Whether patients with lung cancer and EGFR mutation can benefit from anti-PD-1/PD-L1 therapy remains controversial (6).

Several phase 3 clinical trial subgroup analyses show that patients with EGFR-mutated lung cancer fail to receive a prolonged progression-free (PFS) or overall survival benefit from the anti-PD-1/PD-L1 therapy compared with docetaxel $(2,7,8)$. The pooled and retrospective analyses confirm that the EGFR-mutated lung cancer cannot benefit from anti-PD-1/PD-L1 monotherapy (9-12). In a phase 2 clinical study with durvalumab as third- or lateline treatment for advanced EGFR-mutant lung cancer, the clinical activity supports previous reports with median a PFS of 1.9 month (13). In a phase 2 clinical trial, none of the naïve patients with EGFR-mutated tyrosine kinase inhibitor (TKI) and PD-L1 expression $\geq 50 \%$ can respond to the anti-PD-1 therapy (14). Hence, patients with EGFRmutated lung cancer show lack of efficacy to the anti-PD-1/ PD-L1 monotherapy, especially for naïve patients with EGFR-TKI treatment.

Although patients with EGFR-mutated lung cancer show poor response to anti-PD-1/PD-L1 monotherapy, the subgroup analysis of the IMpower 150 trial shows that they can benefit from the combination of atezolizumab, carboplatin, paclitaxel, and bevacizumab (15). This information provides guidance on the immunotherapy in patients with the EGFR-mutated lung cancer, but further confirmation is needed. Several phase 3 clinical trials (i.e., KEYNOTE 789, CheckMate 722, and ORIENT-31) are ongoing to assess the efficacy of the anti-PD-1/PDL1 combined with chemotherapy on patients with EGFR mutation after the failure of target therapy. Therefore, whether patients with EGFR-mutated lung cancer can benefit from immunotherapy combined the chemotherapy and/or bevacizumab after the exhaustion of target therapy remains unknown.

This study aims to analyze the efficacy and safety of anti-PD-1 antibody combined with chemotherapy or/ and bevacizumab therapy in heavily treated patients with EGFR-mutated lung cancer. In this retrospective study, we evaluated the activity of the PD-1 inhibitor combined with other agents (i.e., chemotherapy and/or bevacizumab) in 56 patients with EGFR-mutated lung cancer whose condition have progressed under EGFR-TKI treatment. We also determined which patients can easily benefit from this combination treatment.

We present the following article in accordance with the STROBE reporting checklist (available at http://dx.doi. org/10.21037/jtd-20-3520).

\section{Methods}

\section{Patients}

We reviewed the medical records of all patients with EGFR-mutant positive advanced lung cancer treated at the Jiangsu Cancer Hospital between March 2018 and December 2019 and identified patients who received the PD-1 inhibitor alone or in combination with chemotherapy and/or bevacizumab therapy during the course of their disease. Only patients who progressed after first or second generation of EGFR-TKI and T790M mutation negative subject and patients who carried the T790M mutation and failure after third generation TKI were included in this study. All patients included had at least one measurable disease. The study was conducted in accordance with the Declaration of Helsinki (as revised in 2013). The study was approved by the Academic Ethics Committee of Jiangsu Cancer Hospital. (NO. (2019)160) and individual consent for this retrospective analysis was waived.

\section{Data collection and response assessment}

Medical records were reviewed and extracted on clinical pathologic features and treatment histories. Data and follow-up records were updated as of April 2019. The best response to $\mathrm{PD}-1$ inhibitor-based therapy, defined as a complete or partial response and stable disease achieved at least once during the course of therapy, was assessed using the RECIST v 1.1 criteria. The PFS was defined from the time of treatment initiation to clinical or radiographic progression or death. Adverse events (AEs) were graded in 
accordance with the National Cancer Institute Common Terminology Criteria for Adverse Events v4.0.

\section{Statistical analysis}

Survival data were estimated using the Kaplan-Meier method or Cox survival regression model and compared using the log-rank test in overall cohort and other subgroups. The overall response rates (ORRs) of different subgroups were compared using chi-square test. Statistical analyses were performed using the SPSS version 22.0 (SPSS, Inc.). $\mathrm{P} \leq 0.05$ was considered to indicate statistical significance.

\section{Results}

\section{Patient characteristics}

We identified 56 patients with EGFR mutations at Jiangsu Cancer Hospital who were treated with the PD-1 inhibitor alone or in combination with chemotherapy and/ or bevacizumab therapy. Baseline clinical and pathologic features were summarized in Table 1 . The majority of patients $(92.9 \%, 52 / 56)$ was diagnosed with lung adenocarcinoma. Most EGFR mutation types were EGFR 19 deletion and L858R. An exon 18 mutation and exon 20 insertion were observed. Most (87.5\%) patients had an ECOG performance status of 0 or 1.

\section{Treatment characteristics}

As shown in Table 1, most $(78.6 \%, 44 / 56)$ patients received the PD-1 inhibitor-based therapy as third- or late-line therapy, indicating that they were heavily treated before. We divided patients into four groups in accordance with the different agents that were used to combine with the PD-1 inhibitor (Table 1). Seven patients received PD-1 inhibitor monotherapy. Exactly 21 patients received the PD-1 antibody combined with chemotherapy. Eight patients received the PD-1 antibody combined with bevacizumab. Twenty patients received the PD-1 antibody combined with chemotherapy and bevacizumab. The PD-1 inhibitors used in this study were pembrolizumab, nivolumab, camrelizumab, toripalimab, and sintilimab. Camrelizumab, toripalimab, and sintilimab were PD-1 inhibitors will clinical approval from the Chinese Food and Drug Administration. Besides chemotherapy and bevacizumab therapy, some $(14.3 \%, 8 / 56)$ patients received radiotherapy during the PD-1 inhibitor-based therapy.

\section{Overall clinical outcomes}

As shown in Figure 1A, ORRs were observed in 6 of 56 $(10.7 \%)$ patients, and the disease control rate (DCR) was 53.6\% (30/56). No patient achieved a complete response. The median PFS was 3.33 months with $95 \%$ CI of 1.58 5.08 months (Figure 1B). All six patients that achieved PR were treated with the PD-1 inhibitor combined with chemotherapy or bevacizumab therapy. Three of six patients with PR were treated with radiotherapy combined with PD-1 inhibitor-based therapy. Notably, five patients $(5 / 56,8.9 \%)$ died because of rapid progression within three months after PD-1 inhibitor-based therapy.

\section{Subgroup analyses}

As shown in Table 1 and Figure $2 A$, the ORR of patients who were treated with radiotherapy combined with PD-1 inhibitor-based therapy was significantly higher than that without radiotherapy $(37.6 \%$ vs. $6.3 \%, \mathrm{P}=0.008)$. In all patients treated with radiotherapy, the lesion evaluated for the efficacy of ICI therapy and irradiation site were observed on the same sites, and the treatment processes are shown in Table 2. None of the seven patients who received the PD-1 inhibitor monotherapy achieved complete or partial response. Patients with good performance had higher ORR than those with poor performance. However, no statistical difference was observed possibly because of the limited number of patients (Figure 2B). Patients treated with the PD-1 inhibitor-based therapy as second-line therapy showed relatively higher ORR and longer PFS than those treated with the PD-1 inhibitor-based therapy as third- or lateline therapy (ORR: $25.0 \%$ vs. $6.82 \%, \mathrm{P}=0.071$, Figure $2 C$; PFS: 5.50 months vs. 3.27 months, $\mathrm{P}=0.301$, Figure $3 A$ ).

In multivariate analysis, the most important factors for PFS include combination therapy and ECOG performance status, as shown in Table 3. No significant difference was observed in the PFS of patients with EGFR 19 del and L858 mutation (Figure $3 B, \mathrm{P}=0.465$ ). The PFS of patients treated with radiotherapy was higher than that without radiotherapy (5.50 months vs. 2.93 months, $\mathrm{P}=0.153$, Figure 3C). No significant difference was observed in the ORR, DCR, or PFS under treatment with PD-1 antibody alone, PD-1 antibody combined with chemotherapy, PD-1 antibody combined with bevacizumab, and PD-1 antibody combined with chemotherapy and bevacizumab (Figure 3D) 
Table 1 Clinical characteristics of patients and clinical activity of anti-PD-1 therapy

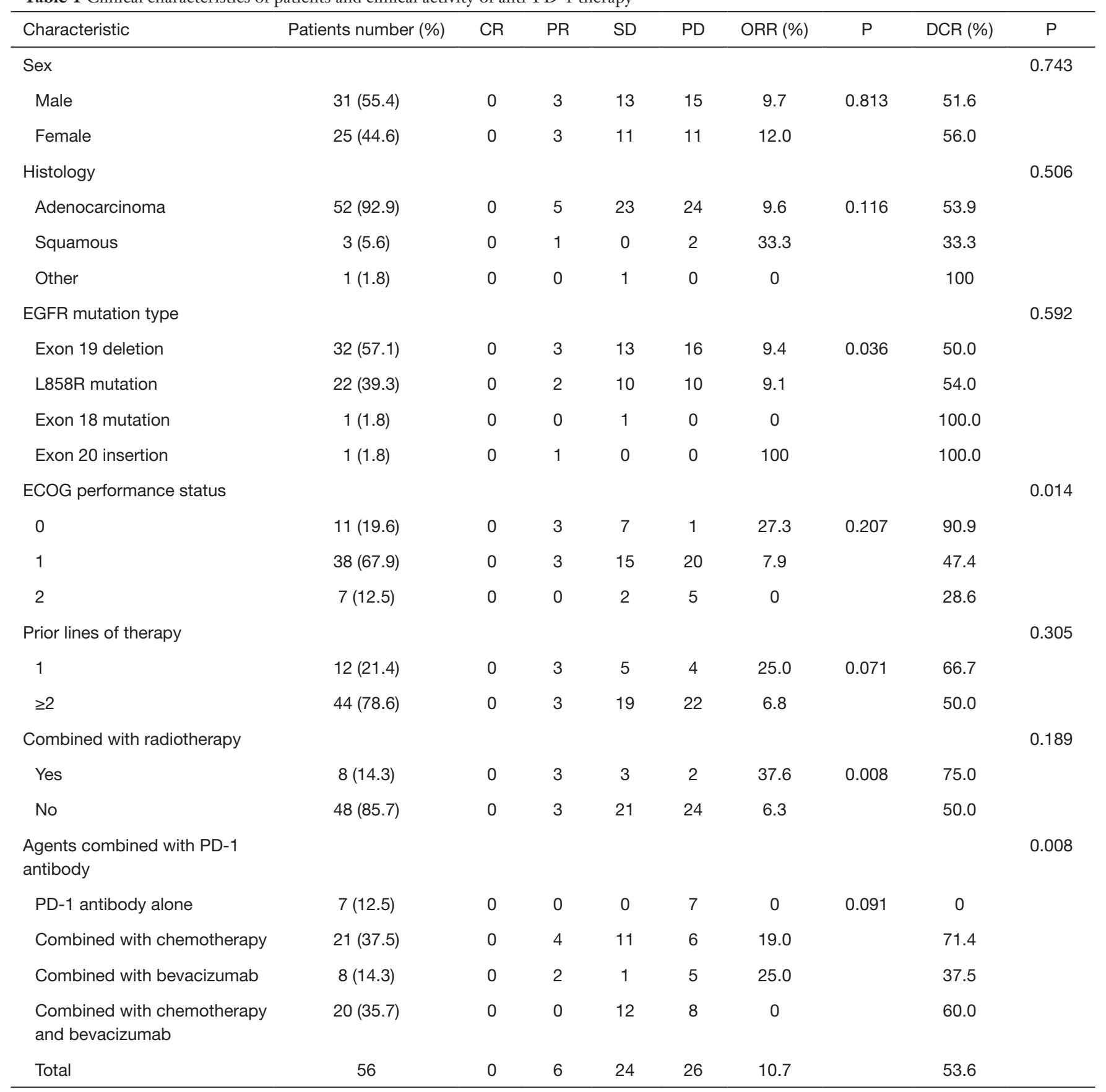

PD-1, programmed death-1; CR, complete response; PR, partial response; SD, stable disease; PD, progressive disease; ORR, objective response rate; DCR, disease control rate.

\section{Safety}

As shown in Table 4, 87.5\% (49/56) of patients experienced treatment-related AEs. Several (42.9\%, 24/56) patients experienced grade 3 or 4 treatment-related AE. One patient discontinued therapy because of grade 3 pneumonitis, which was related to anti-PD-1 therapy. The most common treatment-related AEs were leukopenia, anemia, elevated ALT or AST, fatigue, and decreased appetite. No grade 5 treatment-related $\mathrm{AE}$ was reported. 
A

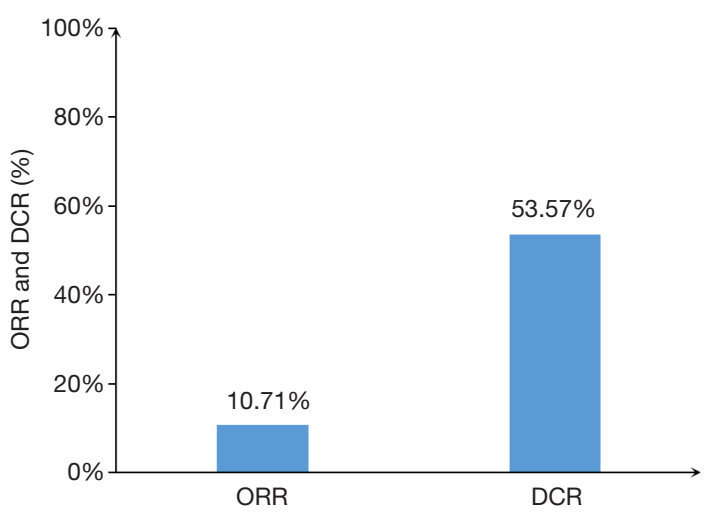

B

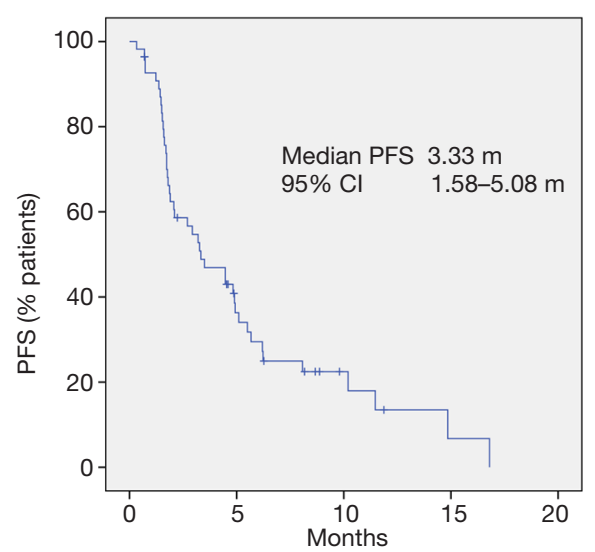

Figure 1 Overall clinical outcomes. (A) Objective response rate (ORR) and disease control rate (DCR) of all patients. (B) Progression-free survival (PFS) of all patients.
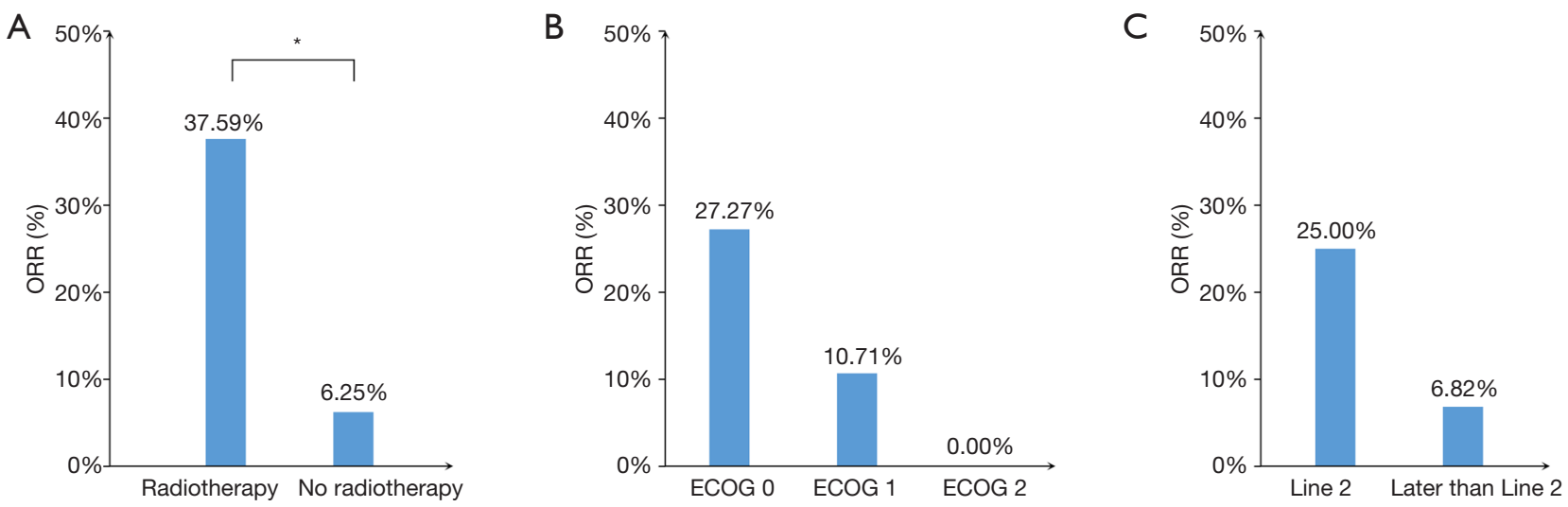

Figure 2 Objective radiographic responses. (A) Objective response rate (ORR) of patients with or without radiotherapy. (B) ORR of patients with different ECOG performance status. (C) ORR of patients treated with ICIs as second-line or later treatment. *, $\mathrm{P}<0.05$.

\section{Discussion}

In this retrospective study, we evaluated the efficacy and safety of the PD-1 inhibitor combined with other therapies on heavily treated patients with advanced lung cancer and EGFR mutation after target therapy. Results show that the PD-1 inhibitor-based combination therapy and the EGFR wild-type lung cancer have similar AE profiles. This combination therapy shows longer PFS than the PD-1 inhibitor monotherapy reported before. However, the addition of chemotherapy and/or bevacizumab therapy to the anti-PD-1 therapy did not remarkably improved on heavily treated patients.

The EGFR-mutated lung cancer was thought to be an uninflamed "cold" tumor with low tumor-infiltrating CD8+
T cells $(16,17)$. The conversion of this "cold" tumor into immunotherapy active "hot" tumor is vital in improving patients' immunotherapy efficacy. The EGFR pathway activation is responsible for the formation of the uninflamed tumor microenvironment $(18,19)$. The treatment of EGFR blockage can modulate key EGFR signaling pathways and increase immune cell infiltration (20), indicating that the treatment of PD-1/PD-L1 inhibitor may have considerable efficacy on EGFR-mutated patients treated with a series of EGFR target therapies. However, the efficacy of the anti-PD-1/PD-L1 monotherapy did not show evident improvement on patients with EGFR mutation who have experienced EGFR-TKI therapy (13). Tumor cells can be killed using chemotherapy and release tumor antigen. These antigens can be presented by dendric cells to $T$ cells 
Table 2 Clinical characteristics, treatment process, clinical activity of patients treated with the radiotherapy

\begin{tabular}{|c|c|c|c|c|c|c|}
\hline Patient & Metastases & Irradiation target & Irradiation area & Irradiation dose (Gy) & Irradiation times (f) & Clinical activity \\
\hline 2 & Brain & PTV & Brain & 45 & 10 & PR \\
\hline 3 & Brain & PTV & Brain & 54 & 20 & SD \\
\hline 4 & Lung & CTV & Lung & 56 & 28 & PR \\
\hline 6 & Lung & PTV & Lung & 56 & 28 & PR \\
\hline 7 & Liver & PTV & Liver & 36 & 6 & PD \\
\hline 8 & Lung & PTV & Lung & 56 & 7 & SD \\
\hline
\end{tabular}

PTV, planning target volume; CTV, clinical target volume; PD, progressive disease; PR, partial response; SD, stable disease.

A

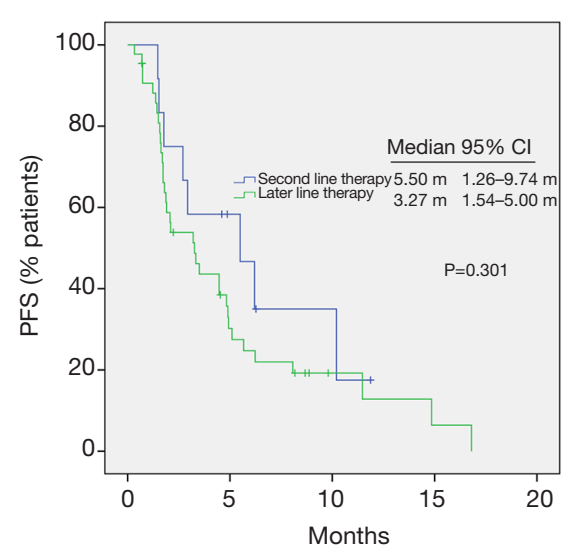

C

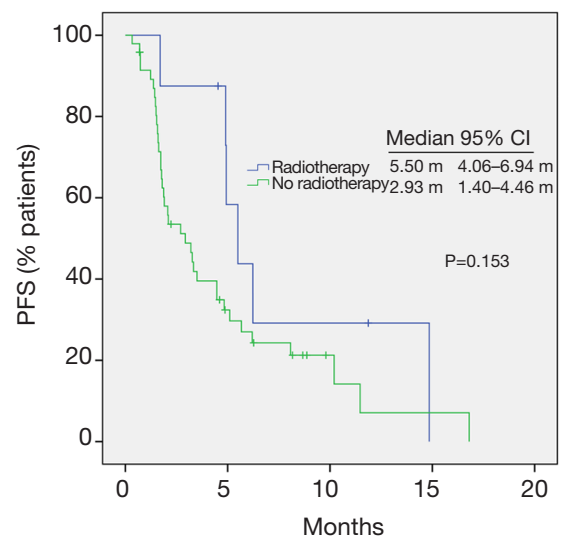

B

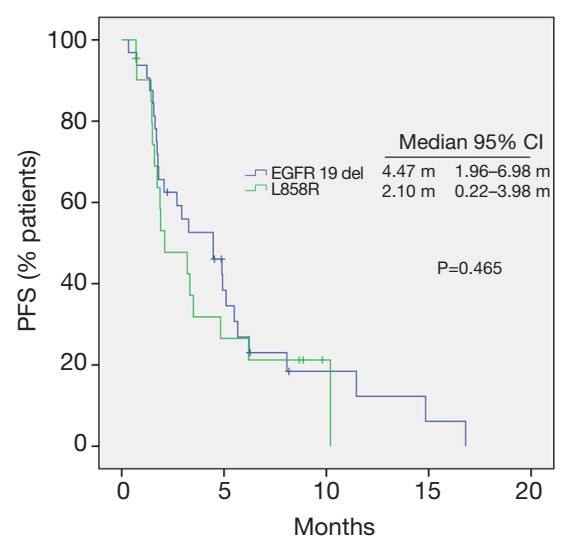

D

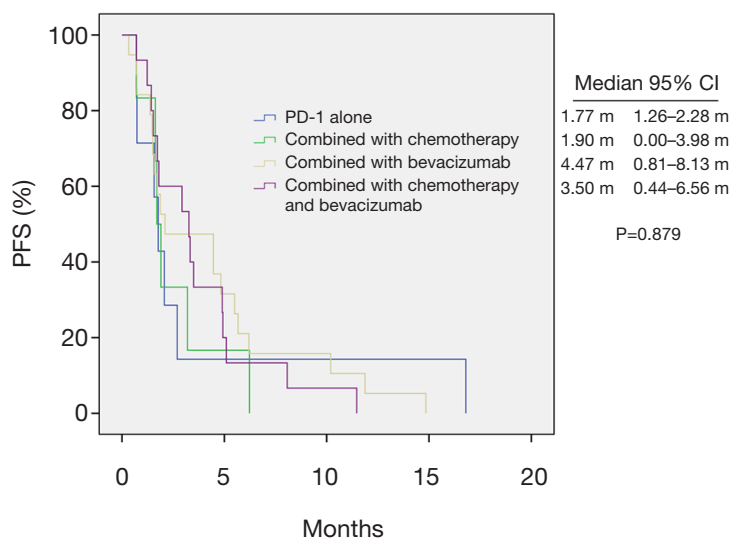

Figure 3 Kaplan-Meier curves. (A) Progression-free survival (PFS) of patients treated with ICIs as second-line or later treatment. (B) PFS of patients with EGFR 19 del and L858 mutation. (C) PFS of patients with or without radiotherapy. (D) PFS of different combination groups. 
Table 3 Univariate analysis and multivariate analysis of factors of progression-free survival

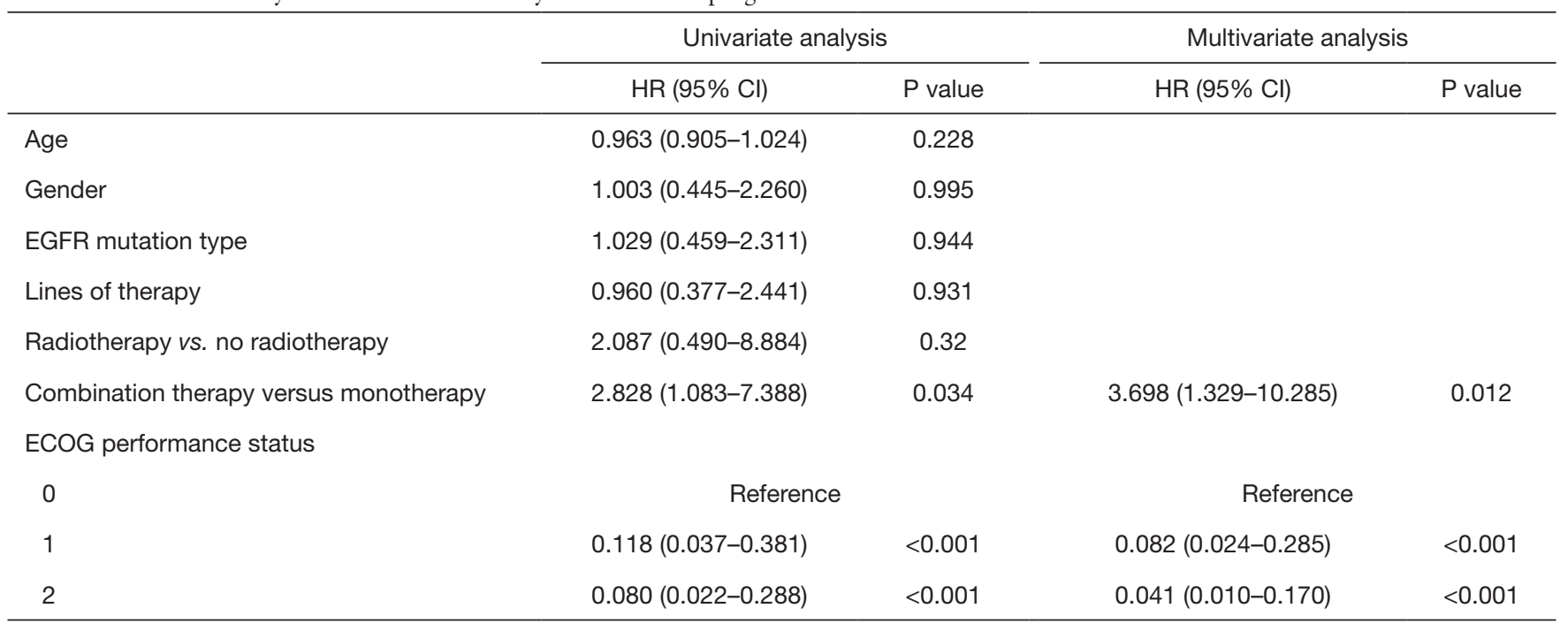

Table 4 Treatment-related adverse events

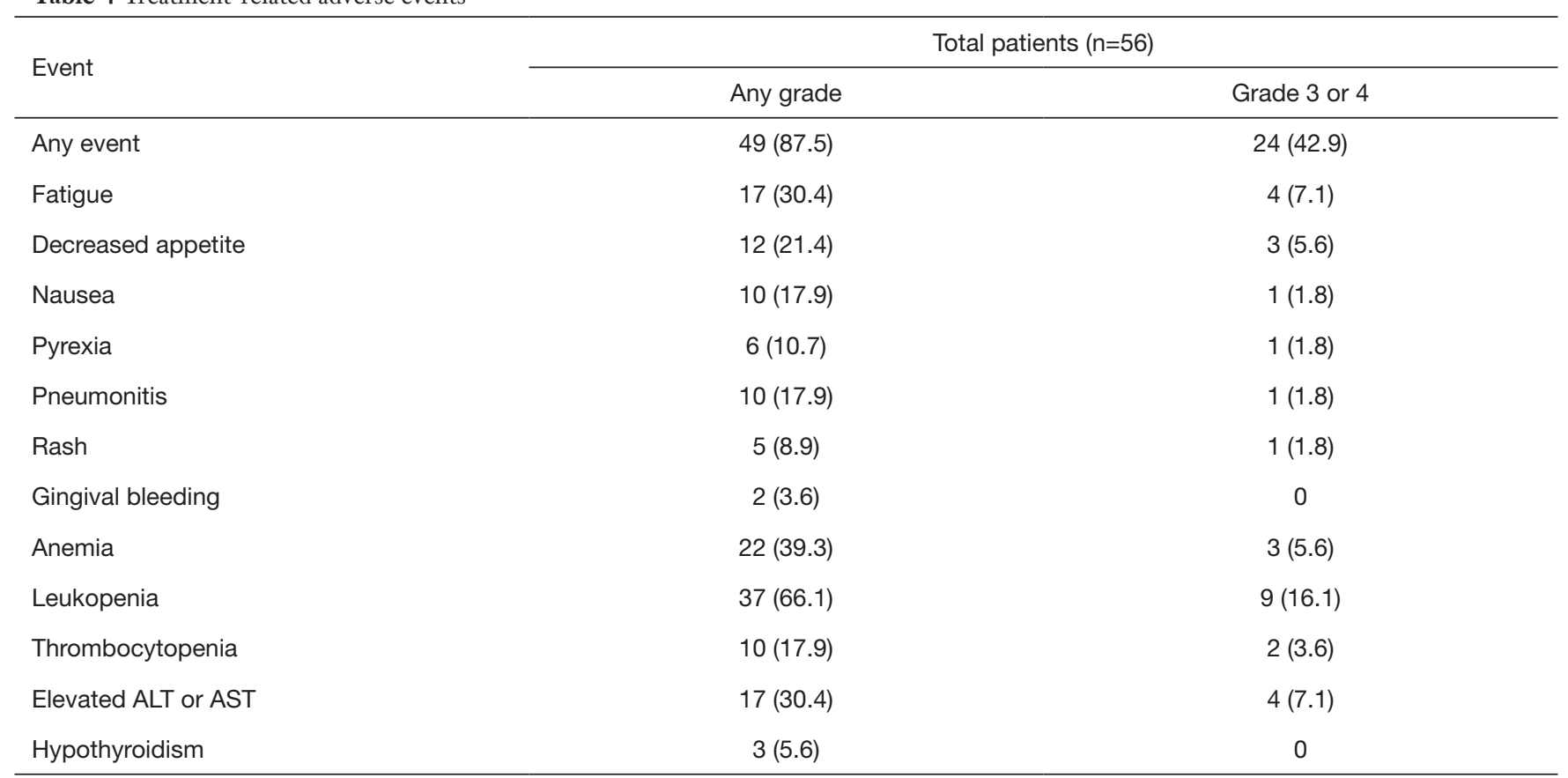

Number of patients with an event (percent). ALT, alanine aminotransferase; AST, aspartate transaminase.

and improve the efficacy of immunotherapy. Therefore, chemotherapy and immunotherapy may have synergistic effects. The combination of the PD-1 inhibitor and chemotherapy may improve the efficacy on patients with EGFR mutation after target therapy. Our study first proves that the combined therapy may result in better response than anti-PD-1 monotherapy but did not show remarkable efficacy on patients with EGFR mutation.

The median PFS is approximately two months for patients with EGFR mutation treated with PD-1/PD-L1 monotherapy $(11,12)$. In our study, the median PFS was 3.3 months and was improved compared with PD-1/PD-L1 monotherapy. However, this improvement is not dramatic, possibly because most patients are heavily treated, and some 
patients have poor performance status before they start to receive the PD-1 inhibitor-based therapy. We performed subgroup analysis and found that patients with good performance status, who received the PD-1 inhibitor-based therapy as second-line therapy or who were treated with the radiotherapy during the treatment of immunotherapy, may show good response. The results will determine which patients are more likely to benefit from the PD-1 inhibitorbased treatment. However, this trend did not have statistical difference possibly because of the limited number of patients included in this study. The high PD-L1 expression on tumor tissues indicates improved response to anti-PD-1 therapy. However, only the PD-L1 expression levels of 10 patients are available in this study. Therefore, we have not determined the efficacy difference at different PD-L1 expression levels. Considering that most of the patients' gene mutation status is not available in this study, we have not analyzed the association between the efficacy and certain gene mutation.

In the 2019 World Conference on Lung Cancer, the result of a phase 2 study of toripalimab, a PD-1 monoclonal antibody, in combination with the chemotherapy in patients with EGFR-positive advanced lung cancer have failed to prior EGFR-TKI therapies. Forty patients were included in this study (21). This study had a relatively high response rate of the PD-1 inhibitor-based therapy on patients with EGFR mutation $(\mathrm{ORR}=50.0 \%, \mathrm{DCR}=87.5 \%)$. The median duration of the response is 7.0 months. The results of the clinical trial are superior to our study, possibly because the patient compositions of these two studies are different. In their study, $97.5 \%$ of patients have received the combination therapy as second-line therapy, and only one patient has received combination therapy as thirdline therapy. Besides, the ECOG performance status of all patients in their study is 0 or 1 . However, in our study, only $21.4 \%$ of patients have received combination therapy as second-line therapy. Most patients have received anti-PD-1 therapy as third- or even late-line therapy. Approximately $12.5 \%$ of patients have poor performance status. The difference in patient composition may contribute to the difference in results of these two studies. Notably, these studies involved limited number of patients, which may result in statistical bias. Therefore, further randomized controlled phase 3 study is needed to confirm whether patients with EGFR-mutated lung cancer can benefit from PD-1 inhibitor-based combination therapy.

In summary, our study proves that the PD-1 inhibitor therapy combined with chemotherapy and/or bevacizumab therapy shows acceptable toxicity profile and moderate efficacy on heavily treated patients with advanced EGFRmutated lung cancer after target therapy.

\section{Acknowledgments}

Funding: This work was supported by the National Natural Science Foundation of China [81871873], the Project of Invigorating Health Care through Science, Technology and Education, Jiangsu Provincial Medical Youth Talent [QNRC2016646], China Postdoctoral Science Foundation [2017M621680], six talent peaks project in Jiangsu Province (WSN-039), and the talents program of Jiangsu Cancer Hospital (YC201807).

\section{Footnote}

Reporting Checklist: The authors have completed the STROBE reporting checklist. Available at http://dx.doi. org/10.21037/jtd-20-3520

Data Sharing Statement: Available at http://dx.doi. org/10.21037/jtd-20-3520

Conflicts of Interest: All authors have completed the ICMJE uniform disclosure form (available at http://dx.doi. org/10.21037/jtd-20-3520). The authors have no conflicts of interest to declare.

Ethical Statement: The authors are accountable for all aspects of the work in ensuring that questions related to the accuracy or integrity of any part of the work are appropriately investigated and resolved. The study was conducted in accordance with the Declaration of Helsinki (as revised in 2013). The study was approved by the Academic Ethics Committee of Jiangsu Cancer Hospital. (NO. (2019)160) and individual consent for this retrospective analysis was waived.

Open Access Statement: This is an Open Access article distributed in accordance with the Creative Commons Attribution-NonCommercial-NoDerivs 4.0 International License (CC BY-NC-ND 4.0), which permits the noncommercial replication and distribution of the article with the strict proviso that no changes or edits are made and the original work is properly cited (including links to both the formal publication through the relevant DOI and the license). See: https://creativecommons.org/licenses/by-nc-nd/4.0/. 


\section{References}

1. Gandhi L, Rodriguez-Abreu D, Gadgeel S, et al. Pembrolizumab plus Chemotherapy in Metastatic NonSmall-Cell Lung Cancer. N Engl J Med 2018;378:2078-92.

2. Borghaei H, Paz-Ares L, Horn L, et al. Nivolumab versus Docetaxel in Advanced Nonsquamous Non-Small-Cell Lung Cancer. N Engl J Med 2015;373:1627-39.

3. Paz-Ares L, Luft A, Vicente D, et al. Pembrolizumab plus Chemotherapy for Squamous Non-Small-Cell Lung Cancer. N Engl J Med 2018;379:2040-51.

4. Zhou C, Wu YL, Chen G, et al. Erlotinib versus chemotherapy as first-line treatment for patients with advanced EGFR mutation-positive non-small-cell lung cancer (OPTIMAL, CTONG-0802): a multicentre, open-label, randomised, phase 3 study. Lancet Oncol 2011;12:735-42.

5. Soria JC, Ohe Y, Vansteenkiste J, et al. Osimertinib in Untreated EGFR-Mutated Advanced Non-Small-Cell Lung Cancer. N Engl J Med 2018;378:113-25.

6. Yu S, Liu D, Shen B, et al.Immunotherapy strategy of EGFR mutant lung cancer. Am J Cancer Res 2018;8:2106-15.

7. Rittmeyer A, Barlesi F, Waterkamp D, et al. Atezolizumab versus docetaxel in patients with previously treated non-smallcell lung cancer (OAK): a phase 3, open-label, multicentre randomised controlled trial. Lancet 2017;389:255-65.

8. Herbst RS, Baas P, Kim DW, et al. Pembrolizumab versus docetaxel for previously treated, PD-L1-positive, advanced non-small-cell lung cancer (KEYNOTE-010): a randomised controlled trial. Lancet 2016;387:1540-50.

9. Lee CK, Man J, Lord S, et al. Checkpoint Inhibitors in Metastatic EGFR-Mutated Non-Small Cell Lung Cancer-A Meta-Analysis. J Thorac Oncol 2017;12:403-7.

10. Lee CK, Man J, Lord S, et al. Clinical and Molecular Characteristics Associated With Survival Among Patients Treated With Checkpoint Inhibitors for Advanced NonSmall Cell Lung Carcinoma: A Systematic Review and Meta-analysis. JAMA Oncol 2018;4:210-6.

11. Gainor JF, Shaw AT, Sequist LV, et al. EGFR Mutations and ALK Rearrangements Are Associated with Low Response Rates to PD-1 Pathway Blockade in Non-Small Cell Lung Cancer: A Retrospective Analysis. Clin Cancer Res 2016;22:4585-93.

12. Mazieres J, Drilon A, Lusque A, et al. Immune checkpoint inhibitors for patients with advanced lung cancer and oncogenic driver alterations: results from the IMMUNOTARGET registry. Ann Oncol
2019;30:1321-8.

13. Garassino MC, Cho BC, Kim JH, et al. Durvalumab as third-line or later treatment for advanced non-small-cell lung cancer (ATLANTIC): an open-label, single-arm, phase 2 study. Lancet Oncol 2018;19:521-36.

14. Lisberg A, Cummings A, Goldman JW, et al. A Phase II Study of Pembrolizumab in EGFR-Mutant, PD-L1+, Tyrosine Kinase Inhibitor Naive Patients With Advanced NSCLC. J Thorac Oncol 2018;13:1138-45.

15. Socinski MA, Jotte RM, Cappuzzo F, et al. Atezolizumab for First-Line Treatment of Metastatic Nonsquamous NSCLC. N Engl J Med 2018;378:2288-301.

16. Dong ZY, Zhang JT, et al. EGFR mutation correlates with uninflamed phenotype and weak immunogenicity, causing impaired response to PD-1 blockade in non-small cell lung cancer. Oncoimmunology 2017;6:e1356145.

17. Liu SY, Dong ZY, Wu SP, et al. Clinical relevance of PD$\mathrm{L} 1$ expression and CD8+ T cells infiltration in patients with EGFR-mutated and ALK-rearranged lung cancer. Lung Cancer 2018;125:86-92.

18. Yu S, Sha H, Qin X, et al. EGFR E746-A750 deletion in lung cancer represses antitumor immunity through the exosome-mediated inhibition of dendritic cells. Oncogene 2020;39:2643-57.

19. Mascia F, Schloemann DT, Cataisson C, et al. Cell autonomous or systemic EGFR blockade alters the immune-environment in squamous cell carcinomas. Int J Cancer 2016;139:2593-7.

20. Thress KS, Jacobs V, Angell HK, et al. Modulation of Biomarker Expression by Osimertinib: Results of the Paired Tumor Biopsy Cohorts of the AURA Phase I Trial. J Thorac Oncol 2017;12:1588-94.

21. Zhang J, Zhou C, Zhao Y, et al. MA11.06 A PII Study of Toripalimab, a PD-1 mAb, in Combination with Chemotherapy in EGFR+ Advanced NSCLC Patients Failed to Prior EGFR TKI Therapies. J Thorac Oncol 2019; 14:S292.

Cite this article as: $\mathrm{Hu} \mathrm{R}$, Zhao Z, Shi Y, Shi M, Xia G, Yu S, Feng J. Immune checkpoint inhibitors combined with chemotherapy/bevacizumab therapy for patients with advanced lung cancer and heavily treated with EGFR mutation: a retrospective analysis. J Thorac Dis 2021;13(5):2959-2967. doi: $10.21037 /$ jtd-20-3520 